FROM THE EDITOR

\title{
What can we learn from the death of Terri Schiavo?
}

Terri Schiavo died on March 31, 2005, at the age of 41 . Virtually thousands of others died or lay dying on that day throughout the world, yet the death of Terri Schiavo gripped not only the attention of the media throughout the United States and much of the world, but the attention of the U.S. Congress, the U.S. President, the Vatican, and millions in the United States and around the world. Why? Well, in the words of U.S. President George Bush, "The case of Terri Schiavo raises complex issues.... Those who live at the mercy of others deserve our special care and concern. It should be our goal as a nation to build a culture of life, where all Americans are valued, welcomed, and protected-and that culture of life must extend to individuals with disabilities" (The New York Times, March 31, 2005). Terri Schiavo, in her persistent vegetative state of 15 years duration, was being kept alive, in her Florida hospice bed, with the help of a feeding tube that artificially delivered fluids and nutrition. The attempts of her husband over the last 7 years, in opposition to the wishes of his wife's parents, to remove the feeding tube and allow his wife to die have created a firestorm of controversy and debate in judicial, medical, political, ethical, moral, and religious arenas. When Terri Schiavo died, some 13 days after the feeding tube was removed, the noted civil rights activist Reverend Jesse Jackson said, "She was starved and dehydrated to death!" (The New York Times, March 31, 2005). A Vatican spokesman said "Exceptions cannot be allowed to the principle of the sacredness of life from conception to its natural death" (The New York Times, March 31, 2005). Clearly, the death of Terri Schiavo rekindled a variety of debates that were perhaps dormant but unresolved. The political debate in the United States and the appropriateness of steps taken by the U.S. President and Congress will likely continue through the next cycle of elections and the process of selecting and approving judicial nominations. They will also, undoubtedly, influence sev- eral aspects of medical research and practice including end-of-life care. The religious and moral debates regarding the sanctity of life will continue and also significantly impact on medical research and medical practice. For those interested in reading more about these particular issues I refer you to two excellent pieces in the April 21, 2005, issue of the New England Journal of Medicine (i.e., Annas, 2005; Quill, 2005). For clinicians and researchers in palliative care, however, the death of Terri Schiavo has raised some rather specific clinical and research issues that must be addressed. These issues pertain primarily to the experience of suffering in the dying process.

Let me briefly recount the medical facts related to the saga of Terri Schiavo's dying and death. On February 25, 1990, Terri Schiavo had a cardiac arrest due to hypokalemia that resulted from an eating disorder (i.e., bulimia and self-induced vomiting). As a result of sever hypoxia and ischemic damage to her brain, Ms. Schiavo fell into a persistent vegetative state and exhibited no evidence of higher cortical functioning. Computed tomographic scans of her brain showed severe atrophy of her cerebral cortex. Electroencephalograms indicated no activity of the cerebral cortex. Her neurologic examination indicated that she had intact brain stem function, but no cortical function. This was all consistent with a diagnosis of persistent vegetative state. Ms. Schiavo required artificially delivered fluids and nutrition, through the use of a feeding tube, to remain alive.

The issue of whether Ms. Schiavo could experience "suffering" during the period of time when she was being "kept alive artificially in a persistent vegetative state" has been raised, particularly by proponents of the right to have medical treatments such as a feeding tube removed in situations where they only prolong the dying process (perhaps also prolonging suffering). As Timothy Quill wrote in his essay entitled, "Terri Schiavo-A Tragedy Com- 
pounded" (Quill, 2005), "There is no evidence that Ms. Schiavo was suffering, since the usual definition of this term requires conscious awareness that is impossible in the absence of cortical activity." The medical consensus appears to be that a human being cannot experience suffering without a functioning cortex. The absence of a functioning cortex does not allow a human being to experience touch, pain, or emotions in the way those of us with functioning cortexes experience these simple and complex emotions. Likely this is true. We could not perform surgery unless this was true. But the question remains, "How much cortical activity is necessary to experience pain and/or suffering?" Clearly most of the patients we care for at the end of life are not in persistent vegetative states, nor do they exhibit a complete lack of cortical activity. What is the experience of the vast majority of our patients who are on ventilators or feeding tubes in a demented or delirious or sedated state? Are they experiencing distress? Are they suffering? Little research has been conducted to answer these questions. I would highlight one study we conducted in hospitalized cancer patients with delirium that showed that over $95 \%$ of patients who recovered from a delirious episode and could remember their experience during the delirious episode experienced that episode as extremely distressing (Breitbart et al., 2002). In delirium, the cortex of the brain is quite dysfunctional, yet even in such a state patients experience distress and suffer.

The issue of whether Ms. Schiavo suffered during the 13 days after her feeding tube was removed has been raised as well. Certainly there were claims that she was being painfully starved and dehydrated to death. Many palliative care practitioners and researchers were quoted in the media, reassuring the public that dying from removal of a feeding tube, and the resulting dehydration and lack of nutrition, was, in fact, a painless and humane process. This, of course, is quite likely true in the case of Ms. Schiavo, who had limited ability to experience suffering because of her lack of cortical activity. Dr. Quill, in fact, reminds us in his essay (Quill, 2005, p. 1630) that "humans died in this way for thousands of years before the advent of feeding tubes." Palliative care clinicians have believed for some time that death from dehydration and lack of food is not painful and is not accompanied by suffering. This belief is based, however, on a research literature that is quite limited and of questionable validity. Shortly after the death of Ms. Schiavo, I conducted a Medline search on the topic "effects of dehydration and withholding of nutrition on the dying or terminal patient." The number of citations retrieved was
100. Of these 100 citations, only a few were empirical studies of the experience of dehydration and withholding of nutrition on dying patients.

One of the most cited studies, supporting the painless nature of dying from dehydration and lack of nutrition, is the NEJM study by Ganzini et al. (2003) in Oregon. This study was a mail survey of hospice nurses who cared for patients who had voluntarily chosen to refuse food and fluids in order to hasten their deaths. In this survey, on a scale of $0-10$ ( 0 being a very bad death, 10 being a very good death), the nurses rated the deaths of these patients as an 8 on average. This study suggests that, at least from the perspective of the observing nurse, dying from dehydration and lack of nutrition results in a "good" death. Clearly, there are limitations to the study, which include a proxy evaluation of the patient experience, bias of which nurses returned the survey (although 72\% did, which is quite acceptable methodologically), and a lack of clarity about what a "good" death actually means. McCann et al. (1994) prospectively studied symptoms of hunger, thirst, and dry mouth experienced by 32 patients in a palliative care setting who were provided with no artificial feeding or hydration. Twenty patients (63\%) experienced no hunger, whereas 11 patients $(34 \%)$ experienced hunger only initially. Similarly, 20 patients $(63 \%)$ experienced either no thirst or thirst only initially. Symptoms of hunger, thirst, and dry mouth could be alleviated in most patients, usually with small amounts of food, fluids, and/or the application of ice chips and lubrication to the lips. Ninety-four percent of the patients received opioids to relieve pain or shortness of breath. Despite the small numbers of patients in this report, we are at least seeing data generated by patients themselves. However, these are not patients in whom feeding tubes are being removed, as in the case of Terri Schiavo. These are also patients who are still capable of taking small amounts of food and fluids to alleviate hunger and thirst.

Several investigators have pointed out that the experience of dying from dehydration and lack of nutrition is, in fact, quite variable from patient to patient and is not uniformly comfortable and without need for careful and selected intervention to control symptoms such as delirium due to dehydration. Morita et al. (2005) demonstrated, in a sample of terminally ill patients with abdominal cancers, that the choices of hydrating or not providing hydration both resulted in symptoms that cause distress. For instance, providing hydration to this patient population could alleviate membranous dehydration signs, but could worsen ascites, peripheral edema, and peripheral edema. Withholding of hydration, however, was associated with symptoms 
of dehydration, thirst, and delirium. Although much of the debate around death from dehydration and lack of nutrition has centered around the symptoms of hunger and thirst, it is becoming more clear that issues such as delirium related to dehydration or adverse effects of hydration also need to be entered into the clinical judgment of the experience of each clinical choice, and also into the clinical management of patients who die with artificial fluids and nutrition withheld or terminated.

What my reading of these studies argues for is more research, of high quality, examining the full range of clinical implications of death with or without hydration or nutrition. I implore our readers and contributors to start to think about these issues anew, particularly from the perspective of studies that could be done to further clarify our understanding of patient experience and to clarify what our message to families and the public should really be regarding the issues raised by the Terri Schiavo case. In the near future, we will be publishing a series of papers stimulated by the Terri Schiavo case. We invite our readers and contributors to submit letters, commentary, reviews, and studies that may shed light on the issues raised.

\section{REFERENCES}

Annas, G.J. (2005). "Culture of Life" politics at the bedside-The case of Terri Schiavo. New England Journal of Medicine, 352, 1710-1715.

Breitbart, W., Gibson, C., \& Tremblay, A. (2002). The delirium experience. Delirium recall and deliriumrelated distress in hospitalized patients with cancer, their spouses/caregivers, and their nurses. Psychosomatics, 43, 183-194.

Ganzini, L., Goy, E.R., Miller, L.L., et al. (2003). Nurses' experience with hospice patients who refuse food and fluids to hasten death. New England Journal of Medicine, 349, 359-365.

McCann, R.M., Hall, W.J., \& Groth-Juncker, A. (1994). Comfort care for terminally ill patients. The appropriate use of nutrition and hydration. Journal of the American Medical Association, 272, 1263-1266.

Morita, T., Hyodo, I., Yoshimi, T., et al. (2005). Association between hydration volume and symptoms in terminally ill cancer patients with abdominal malignancies. Annals of Oncology, 16, 640-647.

Quill, T.E. (2005). Terri Schiavo-A tragedy compounded. New England Journal of Medicine, 352, 1630-1633.

WILLIAM BREITBART, M.D. Editor-in-Chief 